Results The dominant shoulder displayed significantly increased external rotation when compared with the non-dominant $\left(120.92^{\circ} \pm 14.85\right.$ vs $\left.106,78^{\circ} \pm 12,53\right)$. Internal rotation was decreased by $11,99^{\circ}$ in the throwing shoulder $(p=0.047)$. Concerning forward elevation, a tendency for greater values was noted $(p=0.08)$, with a higher degree in the throwing arm.

Conclusions Range of motion was different between shoulders. Our athletes had an increase in external rotation and a loss of internal rotation in the throwing shoulder, being concordant with what is described in other overhead sports. Furthermore, the dominant shoulder had a significant increase in forward elevation. These findings support the need of performing these evaluations to monitor the development of injuries, so that preventive measures can be taken.

\section{THE RELATIONSHIP BETWEEN SHOULDER PAIN PHYSICAL EXAM FINDINGS, AND STRUCTURAL PATHOLOGY IN ELITE WHEELCHAIR ATHLETES}

\begin{abstract}
${ }^{1,8}$ Cheri Blauwet, ${ }^{2,8}$ Wayne Derman, ${ }^{3,8}$ Nick Webborn, ${ }^{4}$ Dylan Morrissey, ${ }^{5} J u l i a n$ Chakraverty, ${ }^{6}$ Paul Martin, ${ }^{7,8}$ Guzel Idrisova. ${ }^{1}$ Harvard Medical School/Spaulding Rehabilitation Hospital, Boston, USA; ${ }^{2}$ University of Stellenbosch/South Africa IOC Injury and IIIness Prevention Reserach Center, Stellenbosch, South Africa; ${ }^{3}$ University of Brighton, Brighton, UK; ${ }^{4}$ Queen Mary Unveristy of London, London, UK; ${ }^{5}$ University Hospitals of Bristol, Bristol, UK; ${ }^{6}$ English Institute of Sport, Manchester, UK; ${ }^{7}$ Russian Paralympic Committee, Moscow, Russian Federation; International Paralympic Committee, Bonn, Germany
\end{abstract}

\subsection{6/bjsports-2021-I0C.82}

Background Although athletes who are wheelchair users for both daily activity and sport participation are at high risk for shoulder injuries, little is known regarding the characteristics of shoulder injury in this population.

Objective To determine the relationship between shoulder symptoms (SS), physical exam findings (PEF), and structural pathology (SP) in elite wheelchair athletes competing in athletics and powerlifting

Design Cross-sectional study.

Setting Three international competitions.

Participants 80 elite wheelchair athletes competing in track $(n=40)$, field $(n=19)$ and powerlifting $(n=20)$ who also used a manual wheelchair for daily mobility.

Assessment of Risk Factors A senior sports physiotherapist and musculoskeletal radiologist obtained measures of SS, PEF, and MSK ultrasound (MSK-U) findings. Relationships between measures and for sub-groups by sporting discipline were calculated. Age, duration of disability, and disability type were evaluated as independent risk factors for pain or structural pathology.

Main Outcome Measurements The Wheelchair Users Shoulder Pain Index (WUSPI), Physical Examination of the Shoulder Scale (PESS), and the ultrasound Shoulder Pathology Rating Scale (USPRS)

Results A total of 51 of 80 athletes reported dominant shoulder pain. PESS scores were $7.4 \pm 6.7$, WUSPI $22.3 \pm$ 26.9 and USPRS $5.2 \pm$ 4.0. A positive main effect was found for pain history on PESS (F1,154 $=9.57 \mathrm{p}=0.002, \eta_{\mathrm{p}}{ }^{2}=$ $0.06)$ but no interaction with athlete sub-group (F2,154 $=1.90$ $\left.\mathrm{p}=0.15, \eta_{\mathrm{p}}{ }^{2}=0.02\right)$. There were no USPRS score differences between sub groups, but track athletes had lower WUSPI scores and lower PESS scores. The WUSPI and PESS which were strongly correlated (0.71), while the USPRS which did not correlate with either the PESS $(0.21)$ or WUSPI $(0.20)$.
Conclusions Elite wheelchair athletes have a high prevalence of MSK-U pathology with low-moderate levels of SS and PEF. MSK-U findings do not correlate with SS or PEF. These findings are an important step to educate the development of targeted preventative measures.

\section{EVALUATION OF SPORT SPECIFIC ADAPTATIONS AT THE SHOULDER JOINT AND CORE ENDURANCE AMONG ELITE FEMALE VOLLEYBALL PLAYERS WITH AND WITHOUT OVERUSE RELATED SHOULDER PROBLEMS}

${ }^{1,2}$ Lydia Bucher, ${ }^{3}$ Pierrette Baschung Pfister, ${ }^{4,5}$ Ann Cools. ${ }^{1}$ Zurich University of Applied Sciences, School of Health Professions, Institute of Physiotherapy, Winterthur, Switzerland; ${ }^{2}$ Schulthess Klinik, Physiotherapy, Zurich, Switzerland; ${ }^{3}$ University Hospital Zurich, Physiotherapy Occupational Therapy Research Center, Zurich, Switzerland; ${ }^{4}$ Ghent University, Department of Rehabilitation Sciences and Physiotherapy, Ghent, Belgium: ${ }^{5}$ University of Copenhagen, Department of Occupational and Physical Therapy and Institute of Sports Medicine, Copenhagen, Denmark

\subsection{6/bjsports-2021-IOC.83}

Background Yet published values outlining the differences of sport-specific adaptations at the shoulder joint between symptomatic (S) and non-symptomatic (nS) overhead athletes vary widely. Information defining the link between overuse injuries in relation to both shoulder joint and core endurance is also lacking.

Objective To evaluate if sport-specific adaptations at the shoulder joint are greater in volleyball players with shoulder problems and core stability would be associated with sportspecific adaptations at the shoulder.

Design Cross-sectional study.

Setting This study was performed during the indoor volleyball season 2017/2018 in coordination with the Swiss Volleyball Federation.

Patients (or Participants) 60 female volleyball players with and without overuse shoulder problems playing in a National League volleyball team.

Interventions (or Assessment of Risk Factors) Standardized clinical field tests for passive shoulder ROM in IR and ER, isometric strength of shoulder IR and ER, scapular dyskinesis test and core endurance test were performed during a test session of $1 \mathrm{~h}$. The assessor was blinded to information on the players' current shoulder status.

Main Outcome Measurements Side, group and subgroup comparisons of ROM, strength, scapular control and core endurance and correlations between core endurance and ER strength deficit, strength ratio ER/IR and scapular dyskinesis were described.

Results All players showed significant adaptations in ROM, strength and scapular control of their dominant shoulder (Ds). Players in the $S$ subgroup had significantly weaker IR strength than nS players (mean difference, $7 \mathrm{~N}$; 95\% CI, 0.54 to 13.05; $P \leq .034 ; r=0.295$ ) and tended to have ER strength deficit. Furthermore, the lower the ER strength deficit, the better the core endurance in the side plank position (Ds: $r=$ $0.30 ; 95 \% \mathrm{CI}, 0.11$ to $0.53 ; P \leq .035)$.

Conclusions Elite female volleyball players showed typical sport-specific adaptations in their dominant shoulder. Values of adaptations did only differ in strength and only between subgroups. Further studies need to quantify the association between core endurance and shoulder strength. 